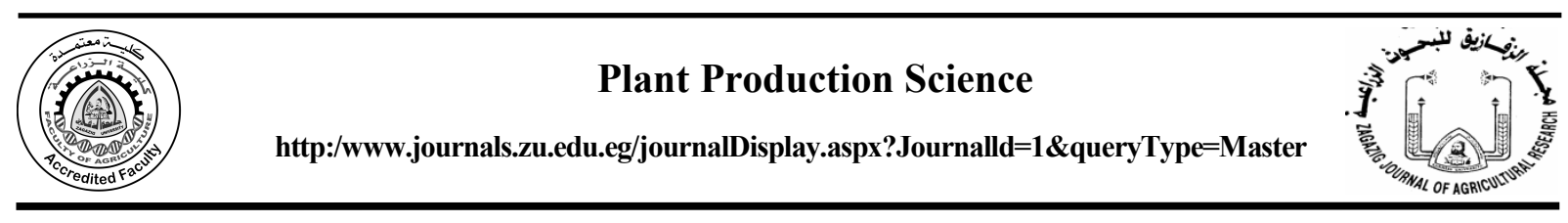

\title{
EFFECT OF PHOSPHORUS FERTILIZATION AND INOCULATION WITH MYCORRHIZAE ON PLANT DRY WEIGHT AND CHEMICAL COMPOSITION OF TWO ONION CULTIVARS
}

\author{
Ahmed Y. Al-Maghraby, A. Bardisi" and A. A.M. Mohsen \\ Hort. Dept., Fac. Agric. Zagazig Univ., Egypt
}

Received: 02/09/2018 ; Accepted: 08/10/2018

\begin{abstract}
One field experiment was carried out during the two successive winter seasons of 2015/2016 and 2016/2017 at a Private Farm in El-Sowa Village, Abo Hammad District, Sharkia Governorate, Egypt, to study the effect of cultivars, mineral phosphorus, and mycorrhizae fungi inoculation on plant dry weight and chemical composition of onion plants under clay soil conditions and flood irrigation system. The experiment included 18 treatments which were the combinations among two onion cultivars (Giza 20 and Beheri improved), three levels of mineral phospours fertilizer $\left(0,30\right.$ and $45 \mathrm{~kg} \mathrm{P}_{2} \mathrm{O}_{5} /$ fad.) and three levels of mycorrhizae $(0,2$ and $4 \mathrm{~kg} /$ fad.). These treatments were arranged in a split split plot design system with three replicates. Cultivars were randomly arranged in the main plots, levels of $\mathrm{P}_{2} \mathrm{O}_{5}$ were randomly distributed in the sub plots, while mycorrhizae levels were randomly arranged in the sub sub plots. The triple interaction treatments among onion Giza 20 cultivar, $\mathrm{P}_{2} \mathrm{O}_{5}$ at $45 \mathrm{~kg} / \mathrm{fad}$., and inoculation with mycorrhizae at $4 \mathrm{~kg} /$ fad., significantly increased dry weight of roots, bulbs, leaves and total dry weight/plant at 75 days after transplanting, as well as, N, P and $\mathrm{K}$ uptake by roots, bulb and leaves at 105 days after transplanting.
\end{abstract}

Key words: Onion, cultivar, $\mathrm{P}_{2} \mathrm{O}_{5}$, mycorrhizae, dry weight, chemical composition.

\section{INTRODUCTION}

Onion (Allium cepa, L.) is one of the oldest and most important vegetable crops gridgen in Egypt for local consumption and exportation. The total cultivated area of onion in Egypt was about 212195 fad., during 2016 season which produced 3115482 tons with average of 14.682 tons/faddan.

Increasing production is one of the national aims in Egyptian agricultural. In this connection, onion production suffered from serious problems that are mostly due to using excess mineral fertilizers such as nitrogen, phosphorus and potassium. These fertilizers, have the major cost in plant production and causes environmental pollution, as well as, contamination under ground water.

There were a significant differences between onion cultivars concerning plant gridgeth
(Muddathir, 2007; Kandil et al., 2013; Abo El-Hamd et al., 2016; Kishor et al., 2017) and plant chemical composition (Mohsen, 2012) on garlic cultivars.

Phosphorus as an important nutritional element plays its part in regulating many physiological criteria in the plant which in turn effect the resulted total yield. The following review of literature of current knowledge on $\mathrm{P}$, may reflect the interest of many workers in studying its mode of action and its role in the productivity of onion. However, one fact must be in mind is that, the provided $\mathrm{P}$ to the plant or the soil depends largely on the available reservation of this element in the soil, so the negative or the positive results may be due to this quantity or sources stored in the soil (Ristimuki et al., 2000).

Increasing phosphorus rate addition from zero to the highest rate to onion plants gave the

\footnotetext{
* Corresponding author: Tel. : +201271887153

E-mail address: abdallabardisi@yahoo.com
} 
highest values of dry weight (Patel and Vachhani, 1994; El-Kalla et al., 1997; ElSheekh, 1997; Mahmoud and El-Hefny, 1999; Mahmoud et al., 2000; Abou El-Magd et al., 2004; El-Desuki, 2004; Shaheen et al., 2007; El-Hamady, 2017) and plant chemical composition (Abd El-Fattah et al., 2002 on garlic; El-Hadidi et al., 2016; Júnior et al., 2016 on onion).

Arbuscular mycorrhizal fungi (AMF) form is a symbiotic association with more than $80 \%$ of land plant families. AMF consists of an internal phase inside the root and an external phase, or extraradical mycelium (ERM) phase, which can form an extensive network within the soil (Gosling et al., 2006). AMF benefit their host principally by increasing uptake of relatively immobile phosphate ions, due to the ability of the fungal to gridge beyond the phosphate depletion zone that quickly develops around the roots (Smith and Read, 1997).

Treated onion plants with mycorrhizae had a significant effect on dry weight of different plant parts (Afek et al., 1991, Bolandnazar, 2009; Shinde and Shinde, 2016 on onion; Wani and Konde, 1998; Sari et al., 2002; Gouda, 2008 on garlic; El-Morsy et al., 2002 on sweet potato) and plant chemical constituents (Mohsen, 2012 on onion; Mohamed, 2015on garlic).

The present work aimed to study the effect of phosphorus and mycorrhizae as a soil inoculation on gridgeth and plant chemical composition of two onion cultivars gridgen under clay soil conditions.

\section{MATERIALS AND METHODS}

One field experiment was carried out during the two successive winter seasons of 2015/2016 and 2016/2017 at a Private Farm in El-Sowa Village, Abo Hammad District, Sharkia Governorate, Egypt, to study the effect of cultivars, mineral phosphorus fertilizer and mycorrhizae colonization on the plant dry weight and chemical composition of onion (Allium cepa L.) under clay soil conditions and flood irrigation system.

The used soil properties were: 52.60 and $54.20 \%$ clay, 24.47 and $23.55 \%$ silt , 22.93 and $22.25 \%$ sand for the two experimental seasons, as well as, 1.54 and $1.52 \%$ organic matter, 7.77 and $7.81 \mathrm{pH}, 1.38$ and $1.35 \mathrm{mmhos} / \mathrm{cm} \mathrm{EC}, 8.62$ and $8.56 \mathrm{ppm}$ available $\mathrm{N}, 0.039$ and 0.034 available $\mathrm{P}$, and 0.55 and 0.53 available $\mathrm{K}$ during the $1^{\text {st }}$ and $2^{\text {nd }}$ seasons, respectively.

This experiment included 18 treatments which were the combination among two onion cultivars (Giza 20 and Behari improved), three rates of mineral phosphorus fertilizer $(0,30$ and $45 \mathrm{~kg} \mathrm{P}_{2} \mathrm{O}_{5} /$ fad.) and three levels of mycorrhizae as soil application $(0,2$ and $4 \mathrm{~kg} / \mathrm{fad}$.)

These treatments were distributed in a split split plots design system with three replications. The onion cultivars were arranged in the main plots, levels of phosphorus fertilizer were randomly distributed in the sub plots, while mycorrhizae levels were randomly distributed in the sub sub plots of the experiment.

The experimental unit area was $12.6 \mathrm{~m}^{2}$. It contains three ridges with $7 \mathrm{~m}$ length each and $60 \mathrm{~cm}$ distance between each two ridges. The seeds of both onion cultivars were obtained from Hort. Res. Inst., Agric. Res. Center, Egypt.

The seeds of onion were sown on 25 and $28^{\text {th }}$ October and transplanted on $20^{\text {th }}$ and $23^{\text {rd }}$ December in the first and second seasons, respectively on both ridges at distance $10 \mathrm{~cm}$ apart.

Mycorrhizae was supplied by Agricultural Microbiology Department, National Research Center, Egypt and it was prepared from $\mathrm{G}$ : Glomus (Indomycorrhizae fungi).

Plants were inoculated with mycorrhizae fungi as a soil application with petmoss as a carrier after successful of onion transplanting.

All plots received $30 \mathrm{~m}^{3} / \mathrm{fad}$., farmyard manure (FYM), $\quad 500 \mathrm{~kg} /$ fad., ammonium sulphate $(20.5 \% \mathrm{~N})$, and $200 \mathrm{~kg} / \mathrm{fad}$., potassium sulphate $\left(48 \% \mathrm{~K}_{2} \mathrm{O}\right)$, one third of $\mathrm{N}, \mathrm{P}$ and $\mathrm{K}$ mineral fertilizers were added during soil preparation, the two thirds of $\mathrm{N}$ and $\mathrm{K}$ were added as soil application at five equal portions every 15 days intervals beginning at 30 days after transplanting, while the two thirds of $\mathrm{P}$ was added at 30, 60 days after transplanting in the form of calcium superphosphate, $15.5 \% \mathrm{P}_{2} \mathrm{O}_{5}$.

The other normal agricultural treatments for gridgeing onion plants, except fertilization treatments were practiced. 


\section{Data Recorded}

Ten plants from each plot were randomly taken at 75 and 105 days after transplanting and the following data were recorded:

\section{Dry weight}

The different parts of onion plant, i.e., roots, bulb and leaves at 75 and 105 days after transplanting were oven dried at $70^{\circ} \mathrm{C}$ till constant weight, then dry weight of roots, bulb, leaves and total dry weight/plant ( $g$ ) were recorded.

\section{Plant chemical composition}

The contents of nitrogen, phosphorus and potassium were assayed in the dry matter from random samples of roots, bulb and leaves at 105 days after transplanting in the second season only. Samples were finely ground and wet digested for $\mathrm{N}, \mathrm{P}$ and $\mathrm{K}$ determination according to the methods advocated by Bremner and Mulvaney (1982), Olsen and Sommers (1982) and Jackson (1970), respectively. After that nitrogen, phosphorus and potassium uptake were estimated, as the dry weight of different parts of onion multiplied by $\mathrm{N}, \mathrm{P}$ and $\mathrm{K}$ contents (\%) in different parts of onion $\mathrm{x} 10$.

\section{Statistical Analysis}

Collected data were subjected to proper statistical analysis of variance according to Snedecor and Cochran (1980), and the differences among treatments were compared using LSD at 0.05 level probability.

\section{RESULTS AND DISCUSSION}

\section{Dry Weight}

\section{Cultivar variation}

There were no significant differences between Giza 20 and Beheri improved cultivars in dry weight of roots, bulb, leaves and total dry weight/plant at 75 and 105 days after transplanting, except dry weight of bulb, leaves and total dry weight/plant at 75 days after transplanting in $1^{\text {st }}$ season only (Table 1).

Some investigators confirmed the differences between onion cultivars on their dry weight (Kandil et al., 2013; Abo El-Hamd et al., 2016; Kishor et al., 2017). In this connection,
Kandil et al. (2010) showed that Giza Red and Giza 20 cultivars significantly increased the dry weight of onion as compared with Beheri.

\section{Effect of phosphorus rates}

Dry weight of roots, bulbs, leaves and total dry weight/plant at 75 and 105 days after transplanting, significantly increased with increasing $\mathrm{P}_{2} \mathrm{O}_{5}$ to $45 \mathrm{~kg} /$ fad., except dry weight of roots at 75 days after transplanting in the $1^{\text {st }}$ season (Table 2).

The superior effect of phosphorus fertilizer application on the dry weight of onion plant is due to that, phosphorus is apart of molecular structure of several vitally important compound, notably nuclic acids (DNA and the two forms of RNA). In addition, phosphorus play indispensable role in photosynthetic, and it is also a constituent of cell nucles and essential for cell division, as well as for the development of meristem tissue (Marchner, 1995)

Such results are connected with those reported by El-Hadidi et al. (2016) and El-Hamady (2017) on onion.

\section{Effect of mycorrhizae colonization levels}

Results in Table 3, show that dry weight of roots, bulbs, leaves and total dry weight/plant at 75 and 105 days after transplanting significantly increased with increasing mycorrhizae levels up to $4 \mathrm{~kg} / \mathrm{fad}$., with insignificant differences with mycorrhizae at $2 \mathrm{~kg} /$ fad., at 105 days after transplanting in both seasons with respect to dry weight of roots.

In this connection, the favourable effect of mycorrhizae on increasing the dry weight of onion plants, is owing directly to their effect on releasing phosphorus in the soil and its absorption by the plant, consequently producing activation energy to utilization of metabolites and building the cells, as well as development of the plant gridgeth (Pacovsky and Fuller, 1986).

Similar results were obtained by other researchers included Tao Guo et al. (2006) who showed that treated onion plants with mycorrhiza colonization resulted in increased shoots dry weight than untreated plants. Also, Shuab et al. (2014) found that dry weight of onion plant was the maximum when plants were infected with mycorrhiza as compared to the uninoculated ones. 
Table 1. Cultivar variation in dry weight of different parts of onion plants at 75 and 105 days after transplanting during $2015 / 2016$ and $2016 / 2017$ seasons

\begin{tabular}{|c|c|c|c|c|c|c|c|c|}
\hline \multirow[t]{3}{*}{ Onion cultivar } & \multicolumn{2}{|c|}{$\begin{array}{l}\text { Dry weight of } \\
\text { roots (g/plant) }\end{array}$} & \multicolumn{2}{|c|}{$\begin{array}{l}\text { Dry weight of } \\
\text { bulb (g/plant) }\end{array}$} & \multicolumn{2}{|c|}{$\begin{array}{l}\text { Dry weight of } \\
\text { leaves (g/plant) }\end{array}$} & \multicolumn{2}{|c|}{$\begin{array}{c}\text { Total dry weight } \\
\text { (g/plant) }\end{array}$} \\
\hline & \multicolumn{8}{|c|}{ Days after transplanting } \\
\hline & 75 & 105 & 75 & 105 & 75 & 105 & 75 & 105 \\
\hline & \multicolumn{8}{|c|}{$2015 / 2016$ season } \\
\hline Giza 20 & 0.57 & 1.26 & 1.95 & 5.94 & 3.18 & 7.29 & 5.41 & 13.85 \\
\hline Beheri improved & 0.61 & 1.24 & 2.75 & 6.05 & 4.43 & 7.42 & 7.49 & 14.09 \\
\hline \multirow[t]{2}{*}{ LSD at 0.05 level } & NS & NS & 0.45 & NS & 0.30 & NS & 0.33 & NS \\
\hline & \multicolumn{8}{|c|}{ 2016/2017 season } \\
\hline Giza 20 & 0.60 & 1.48 & 2.88 & 6.99 & 4.70 & 8.57 & 7.88 & 16.30 \\
\hline Beheri improved & 0.71 & 1.46 & 3.56 & 7.12 & 5.82 & 8.73 & 9.74 & 16.58 \\
\hline LSD at 0.05 level & NS & NS & NS & NS & NS & NS & NS & NS \\
\hline
\end{tabular}

Table 2. Effect of phosphorus rates on dry weight of different parts of onion plants at 75 and 105 days after transplanting during 2015/2016 and 2016/2017 seasons

\begin{tabular}{|c|c|c|c|c|c|c|c|c|}
\hline \multirow[t]{3}{*}{$\overline{\mathrm{P}_{2} \mathrm{O}_{5} \text { rate }(\mathrm{kg} / \mathrm{fad} .)}$} & \multicolumn{2}{|c|}{$\begin{array}{l}\text { Dry weight of } \\
\text { roots (g/plant) }\end{array}$} & \multicolumn{2}{|c|}{$\begin{array}{l}\text { Dry weight of } \\
\text { bulb (g/plant) }\end{array}$} & \multicolumn{2}{|c|}{$\begin{array}{l}\text { Dry weight of } \\
\text { leaves (g/plant) }\end{array}$} & \multicolumn{2}{|c|}{$\begin{array}{c}\text { Total dry weight } \\
\text { (g/plant) }\end{array}$} \\
\hline & \multicolumn{8}{|c|}{ Days after transplanting } \\
\hline & 75 & 105 & 75 & 105 & 75 & 105 & 75 & 105 \\
\hline & \multicolumn{8}{|c|}{$2015 / 2016$ season } \\
\hline $\mathbf{0}$ & 0.55 & 0.87 & 1.94 & 3.86 & 3.17 & 4.74 & 5.40 & 9.03 \\
\hline 30 & 0.64 & 1.22 & 2.21 & 5.43 & 3.60 & 6.66 & 6.12 & 12.70 \\
\hline 45 & 0.57 & 1.68 & 2.90 & 8.69 & 4.65 & 10.66 & 7.83 & 20.19 \\
\hline \multirow[t]{2}{*}{ LSD at 0.05 level } & NS & 0.17 & 0.48 & 0.99 & 0.56 & 1.24 & 0.99 & 2.24 \\
\hline & \multicolumn{8}{|c|}{$2016 / 2017$ season } \\
\hline $\mathbf{0}$ & 0.48 & 1.02 & 2.08 & 4.54 & 3.39 & 5.57 & 5.71 & 10.63 \\
\hline 30 & 0.67 & 1.41 & 3.42 & 6.39 & 5.58 & 7.84 & 9.33 & 14.94 \\
\hline 45 & 0.82 & 1.98 & 4.17 & 10.22 & 6.81 & 12.54 & 11.39 & 23.75 \\
\hline LSD at 0.05 level & 0.13 & 0.20 & 0.22 & 1.18 & 0.37 & 1.43 & 0.62 & 2.65 \\
\hline
\end{tabular}


Table 3. Effect of mycorrhizae levels on dry weight of different parts of onion at 75 and 105 days after transplanting during 2015/2016 and 2016/2017 seasons

\begin{tabular}{|c|c|c|c|c|c|c|c|c|}
\hline \multirow[t]{3}{*}{$\begin{array}{l}\text { Mycorrhizae levels } \\
\text { (kg/fad.) }\end{array}$} & \multicolumn{2}{|c|}{$\begin{array}{l}\text { Dry weight of } \\
\text { roots (g/plant) }\end{array}$} & \multicolumn{4}{|c|}{$\begin{array}{l}\text { Dry weight of Dry weight of } \\
\text { bulb (g/plant) leaves (g/plant) }\end{array}$} & \multicolumn{2}{|c|}{$\begin{array}{l}\text { Total dry weight } \\
\text { (g/plant) }\end{array}$} \\
\hline & \multicolumn{8}{|c|}{ Days after transplanting } \\
\hline & 75 & 105 & 75 & 105 & 75 & 105 & 75 & 105 \\
\hline & \multicolumn{8}{|c|}{$2015 / 2016$ season } \\
\hline $\mathbf{0}$ & 0.49 & 1.11 & 1.68 & 5.27 & 2.66 & 6.47 & 4.58 & 12.30 \\
\hline 2 & 0.53 & 1.32 & 2.25 & 5.85 & 3.68 & 7.17 & 6.20 & 13.68 \\
\hline 4 & 0.75 & 1.32 & 3.12 & 6.86 & 5.09 & 8.42 & 8.58 & 15.94 \\
\hline \multirow[t]{2}{*}{ LSD at 0.05 level } & 0.11 & 0.14 & 0.38 & 0.46 & 0.51 & 0.57 & 1.82 & 1.05 \\
\hline & \multicolumn{8}{|c|}{$2016 / 2017$ season } \\
\hline $\mathbf{0}$ & 0.60 & 1.30 & 2.82 & 6.20 & 4.60 & 7.61 & 7.72 & 14.47 \\
\hline 2 & 0.65 & 1.55 & 3.22 & 6.88 & 5.26 & 8.44 & 8.80 & 16.10 \\
\hline 4 & 0.73 & 1.56 & 3.63 & 8.07 & 5.91 & 9.91 & 9.91 & 18.76 \\
\hline LSD at 0.05 level & 0.09 & 0.16 & 0.15 & 0.54 & 0.27 & 0.65 & 0.45 & 1.23 \\
\hline
\end{tabular}

Effect of the interaction between cultivars and phosphorus rates

The interaction between onion cultivars and $\mathrm{P}_{2} \mathrm{O}_{5}$ had a significant effect on dry weight of roots, bulb, leaves and total dry weight/plant at 75 and 105 days after transplanting in both seasons, except at 75 days after transplanting in the $1^{\text {st }}$ season with respect to dry weight of roots/plant (Table 4). At 105 days after transplanting, the interaction between Giza 20 and Beheri improved cultivars and $\mathrm{P}_{2} \mathrm{O}_{5}$ at 45 $\mathrm{kg} /$ fad., increased dry weight of roots, bulbs, leaves and total dry weight/plant in both seasons.

\section{Effect of the interaction between cultivars and mycorrhizae colonization levels}

The interaction between cultivars and mycorrhizae had a significant effect on dry weight of roots, bulbs, leaves and total dry weight/plant at 75 and 105 days after transplanting in both seasons (Table 5). Colonization Giza 20 cultivar with mycorrhizae at $4 \mathrm{~kg} / \mathrm{fad}$., increased dry weight of roots, bulb, leaves and total dry weight/plant at 105 days after transplanting in both seasons.
These results are in harmony with those reported by Sharma et al. (2000) they showed that all colonized onion cultivars showed higher values for fresh weight and shoot dry matter than non- inoculated plants.

Effect of the interaction between phosphorus and mycorrhizae colonization levels

The interaction between $\mathrm{P}_{2} \mathrm{O}_{5}$ and mycorrhizae had a significant effect on dry weight of roots, bulbs, leaves and total dry weight/plant at 75 and 105 days after transplanting in both seasons (Table 6). At 105 days after transplanting, the interaction between $\mathrm{P}_{2} \mathrm{O}_{5}$ at $45 \mathrm{~kg} /$ fad., and mycorrhizae at 2 and $4 \mathrm{~kg} / \mathrm{fad}$., increased dry weight of roots, bulbs, leaves and total dry weight/plant in both seasons. This means that $\mathrm{P}_{2} \mathrm{O}_{5}$ at $45 \mathrm{~kg} /$ fad., and mycorrhizae colonization at $2 \mathrm{~kg} / \mathrm{fad}$., increased dry weight of onion plants.

Obtained results are coincided with those mentioned by Singh et al. (2002). They reported that each of fresh and dry weights of bulbs was the highest with the combination between $\mathrm{N}+\mathrm{P}$ $+\mathrm{K}$ and mycorrhizae. Also, Abdullahi and Sheriff (2013) showed that inoculated onion plants 
Table 4. Effect of the interaction between cultivars and phosphorus rates on dry weight of different parts of onion plants at 75 and 105 days after transplanting 2015/2016 and 2016/2017 seasons

\begin{tabular}{|c|c|c|c|c|c|c|c|c|c|}
\hline \multirow[t]{3}{*}{$\overline{\text { Onion cultivar }}$} & \multirow{3}{*}{$\begin{array}{l}\mathrm{P}_{2} \mathrm{O}_{5} \\
\text { rates }\end{array}$} & $\begin{array}{l}\text { Dry v } \\
\text { roots }\end{array}$ & ight of & \multicolumn{2}{|c|}{$\begin{array}{l}\text { Dry weight of } \\
\text { bulb (g/plant) }\end{array}$} & \multicolumn{2}{|c|}{$\begin{array}{l}\text { Dry weight of } \\
\text { leaves (g/plant) }\end{array}$} & \multicolumn{2}{|c|}{$\begin{array}{l}\text { Total dry weight } \\
\text { (g/plant) }\end{array}$} \\
\hline & & \multicolumn{8}{|c|}{ Days after transplanting } \\
\hline & & 75 & 105 & $\overline{75}$ & 105 & 75 & 105 & $\overline{75}$ & 105 \\
\hline & & \multicolumn{8}{|c|}{$2015 / 2016$ season } \\
\hline \multirow[t]{3}{*}{ Giza 20} & $\mathbf{0}$ & 0.48 & 0.72 & 1.76 & 3.42 & 2.88 & 4.20 & 4.88 & 7.99 \\
\hline & 30 & 0.61 & 1.27 & 1.83 & 5.24 & 2.98 & 6.43 & 5.11 & 12.32 \\
\hline & 45 & 0.61 & 1.77 & 2.26 & 9.14 & 3.47 & 11.22 & 6.25 & 21.25 \\
\hline \multirow[t]{3}{*}{ Beheri improved } & $\mathbf{0}$ & 0.63 & 1.01 & 2.13 & 4.30 & 3.68 & 5.27 & 5.91 & 10.07 \\
\hline & 30 & 0.67 & 1.13 & 2.58 & 5.62 & 4.22 & 6.89 & 7.13 & 13.08 \\
\hline & 45 & 0.54 & 1.60 & 3.54 & 8.23 & 5.61 & 10.10 & 9.42 & 19.13 \\
\hline \multirow[t]{2}{*}{ LSD at 0.05 level } & & NS & 0.25 & 0.62 & 1.41 & 0.82 & 1.72 & 1.40 & 3.17 \\
\hline & & \multicolumn{8}{|c|}{$2016 / 2017$ season } \\
\hline \multirow[t]{3}{*}{ Giza 20} & $\mathbf{0}$ & 0.50 & 0.85 & 1.80 & 4.03 & 2.94 & 4.94 & 5.00 & 9.40 \\
\hline & 30 & 0.53 & 1.50 & 3.08 & 6.17 & 5.03 & 7.57 & 8.37 & 14.50 \\
\hline & 45 & 0.78 & 2.08 & 3.76 & 10.76 & 6.13 & 13.20 & 10.28 & 25.00 \\
\hline \multirow[t]{3}{*}{ Beheri improved } & $\mathbf{0}$ & 0.47 & 1.18 & 2.35 & 5.05 & 3.83 & 6.20 & 6.42 & 11.85 \\
\hline & 30 & 0.82 & 1.32 & 3.76 & 6.61 & 6.13 & 8.11 & 10.29 & 15.39 \\
\hline & 45 & 0.86 & 1.87 & 4.59 & 9.68 & 7.49 & 11.89 & 12.50 & 22.51 \\
\hline LSD at 0.05 level & & 0.18 & 0.29 & 0.31 & 1.65 & 0.53 & 2.03 & 0.86 & 3.72 \\
\hline
\end{tabular}

Table 5. Effect of the interaction between cultivars and mycorrhizae levels on dry weight of different parts of onion plants at 75 and 105 days after transplanting 2015/2016 and 2016/2017 seasons

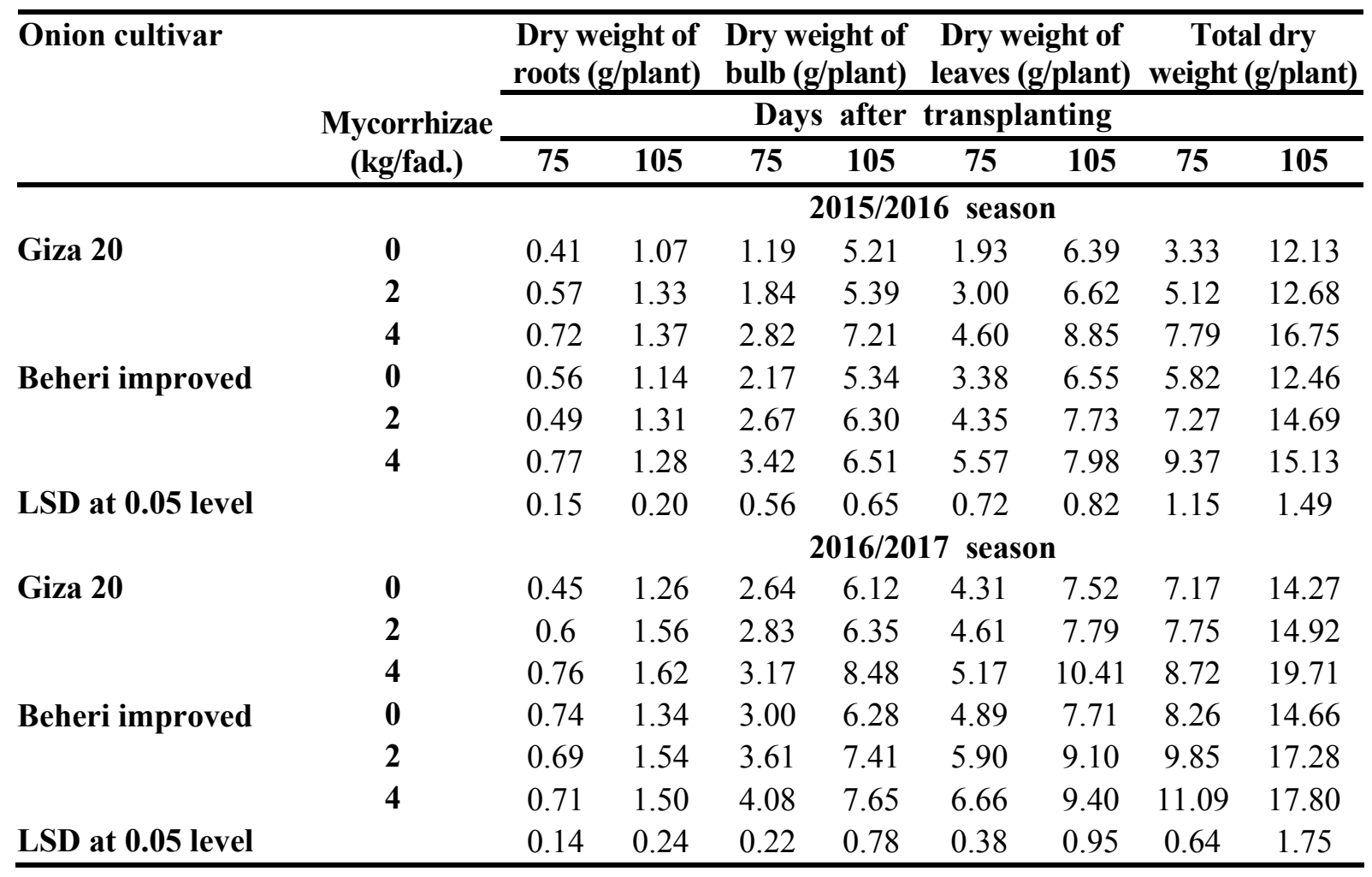


Table 6. Effect of the interaction between phosphorus and mycorrhizae levels on dry weight of different parts of onion plants at 75 and 105 days after transplanting 2015/2016 and 2016/2017 seasons

\begin{tabular}{|c|c|c|c|c|c|c|c|c|c|}
\hline \multicolumn{2}{|l|}{ Treatment } & \multicolumn{2}{|c|}{$\begin{array}{l}\text { Dry weight of } \\
\text { roots (g/plant) }\end{array}$} & \multicolumn{2}{|c|}{$\begin{array}{l}\text { Dry weight of } \\
\text { bulb (g/plant) }\end{array}$} & \multicolumn{2}{|c|}{$\begin{array}{l}\text { Dry weight of } \\
\text { leaves (g/plant) }\end{array}$} & \multicolumn{2}{|c|}{$\begin{array}{l}\text { Total dry weight } \\
\text { (g/plant) }\end{array}$} \\
\hline \multirow{2}{*}{$\begin{array}{c}\mathrm{P}_{2} \mathrm{O}_{5} \text { rates } \\
\text { (kg/fad.) }\end{array}$} & \multirow{2}{*}{$\begin{array}{c}\text { Mycorrhizae } \\
\text { (kg/fad.) }\end{array}$} & \multicolumn{8}{|c|}{ Days after transplanting } \\
\hline & & 75 & 105 & 75 & 105 & 75 & 105 & 75 & 105 \\
\hline \multirow{4}{*}{ 0 } & & & & & $015 / 20$ & 6 seaso & & & \\
\hline & 0 & 0.39 & 0.91 & 1.25 & 3.88 & 2.04 & 4.76 & 3.48 & 9.09 \\
\hline & 2 & 0.38 & 0.96 & 1.74 & 3.40 & 2.84 & 4.18 & 4.77 & 8.06 \\
\hline & 4 & 0.89 & 0.72 & 2.85 & 4.30 & 4.64 & 5.28 & 7.93 & 9.94 \\
\hline \multirow[t]{3}{*}{30} & $\mathbf{0}$ & 0.58 & 0.91 & 1.70 & 4.63 & 2.76 & 5.68 & 4.75 & 10.77 \\
\hline & 2 & 0.62 & 1.28 & 2.17 & 5.14 & 3.53 & 6.30 & 6.01 & 12.08 \\
\hline & 4 & 0.71 & 1.41 & 2.76 & 6.52 & 4.49 & 8.01 & 7.61 & 15.23 \\
\hline \multirow[t]{3}{*}{45} & $\mathbf{0}$ & 0.5 & 1.49 & 2.09 & 7.31 & 3.17 & 8.97 & 5.50 & 17.03 \\
\hline & 2 & 0.59 & 1.72 & 2.85 & 9.00 & 4.65 & 11.04 & 7.80 & 20.90 \\
\hline & 4 & 0.64 & 1.85 & 3.76 & 9.76 & 6.12 & 11.97 & 10.19 & 22.65 \\
\hline \multirow{2}{*}{\multicolumn{2}{|c|}{ LSD at 0.05 level }} & 0.19 & 0.25 & 0.69 & 0.80 & 0.88 & 0.98 & 1.41 & 1.82 \\
\hline & & & & & $016 / 20$ & 7 seaso & & & \\
\hline \multirow[t]{3}{*}{$\mathbf{0}$} & $\mathbf{0}$ & 0.35 & 1.07 & 1.51 & 4.56 & 2.46 & 5.60 & 4.14 & 10.69 \\
\hline & 2 & 0.48 & 1.13 & 2.00 & 4.01 & 3.26 & 4.91 & 5.52 & 9.48 \\
\hline & 4 & 0.61 & 0.85 & 2.72 & 5.06 & 4.44 & 6.21 & 7.47 & 11.70 \\
\hline \multirow[t]{3}{*}{30} & $\mathbf{0}$ & 0.63 & 1.07 & 2.84 & 5.45 & 4.63 & 6.69 & 7.79 & 12.68 \\
\hline & 2 & 0.67 & 1.51 & 3.55 & 6.04 & 5.80 & 7.42 & 9.69 & 14.22 \\
\hline & 4 & 0.72 & 1.65 & 3.86 & 7.67 & 6.30 & 9.42 & 10.53 & 17.92 \\
\hline \multirow[t]{3}{*}{45} & $\mathbf{0}$ & 0.81 & 1.75 & 4.11 & 8.60 & 6.71 & 10.55 & 11.22 & 20.03 \\
\hline & 2 & 0.79 & 2.02 & 4.11 & 10.59 & 6.71 & 13.00 & 11.21 & 24.60 \\
\hline & 4 & 0.87 & 2.17 & 4.29 & 11.48 & 7.00 & 14.08 & 11.73 & 26.64 \\
\hline \multicolumn{2}{|c|}{ LSD at 0.05 level } & 0.17 & 0.29 & 0.27 & 0.86 & 0.47 & 1.16 & 0.79 & 2.14 \\
\hline
\end{tabular}

with mycorrhizal fungi and fertilized with 60 $30-50 \mathrm{~kg} / \mathrm{ha}$ NPK, gave the highest value for each of fresh and dry of shoots than uninoculated plants with high rates (120-60-50 kg/ha NPK).

Effect of the triple interaction among cultivars, phosphorus and mycorrhizae colonization levels

The interaction among cultivars, $\mathrm{P}_{2} \mathrm{O}_{5}$ and mycorrhizae had a significant effect on dry weight of roots, bulbs, leaves and total dry weight/plant at 75 and 105 days after transplanting in both seasons (Tables 7 and 8). The interaction among Giza 20 cultivar, $\mathrm{P}_{2} \mathrm{O}_{5}$ at $45 \mathrm{~kg} /$ fad., and mycorrhizae at 2 or $4 \mathrm{~kg} / \mathrm{fad}$., increased dry weight of roots, bulbs, leaves and total dry weight/plant at 105 days after transplanting in both seasons, with no significant differences with Beheri improved, $\mathrm{P}_{2} \mathrm{O}_{5}$ at $45 \mathrm{~kg} / \mathrm{fad}$., and mycorrhizae at $4 \mathrm{~kg} / \mathrm{fad}$., with respect to total dry weight/plant at 105 days after transplanting.

Nitrogen, Phosphorus and Potassium Uptake

\section{Cultivars variation}

Giza 20 cultivar gave higher N, P and K uptake by roots, bulb and leaves than Beheri improved cultivar at 105 days after transplanting in the second season only (Table 9).

These results are in harmony with those reported by El-Mansi et al. (1984) and Mohsen (2012) 
Table 7. Effect of the triple interaction among cultivars, phosphorus rates and mycorrhizae levels on dry weight of different parts of onion plants at 75 and 105 days after transplanting during 2015/ 2016 season

Treatment

Dry weight of Dry weight of Dry weight of Total dry

roots (g/plant) bulb (g/plant) leaves (g/plant) weight (g/plant)

\begin{tabular}{|c|c|c|c|c|c|c|c|c|c|c|}
\hline Cultivar & $\mathrm{P}_{2} \mathrm{O}_{5}$ rates & Mycorrhizae & & & Day & after & ans! & nting & & \\
\hline & & & 75 & 105 & 75 & 105 & 75 & 105 & 75 & 105 \\
\hline
\end{tabular}

\begin{tabular}{|c|c|c|c|c|c|c|c|c|c|c|}
\hline \multirow[t]{9}{*}{ Giza 20} & \multirow[t]{3}{*}{ 0 } & $\mathbf{0}$ & 0.28 & 0.77 & 1.14 & 3.32 & 1.86 & 4.07 & 3.14 & 7.77 \\
\hline & & 2 & 0.39 & 0.74 & 1.60 & 2.98 & 2.61 & 3.66 & 4.40 & 7.01 \\
\hline & & 4 & 0.78 & 0.67 & 2.55 & 3.98 & 4.17 & 4.88 & 7.11 & 9.19 \\
\hline & \multirow[t]{3}{*}{30} & $\mathbf{0}$ & 0.46 & 0.93 & 1.30 & 4.53 & 2.12 & 5.56 & 3.65 & 10.56 \\
\hline & & 2 & 0.61 & 1.34 & 1.60 & 3.96 & 2.62 & 4.85 & 4.52 & 9.48 \\
\hline & & 4 & 0.76 & 1.55 & 2.58 & 7.24 & 4.20 & 8.90 & 7.17 & 16.92 \\
\hline & \multirow[t]{3}{*}{45} & $\mathbf{0}$ & 0.50 & 1.51 & 1.12 & 7.77 & 1.83 & 9.54 & 3.20 & 18.06 \\
\hline & & 2 & 0.71 & 1.90 & 2.32 & 9.25 & 3.78 & 11.35 & 6.45 & 21.55 \\
\hline & & 4 & 0.63 & 1.90 & 3.34 & 10.42 & 5.45 & 12.77 & 9.09 & 24.14 \\
\hline \multirow[t]{9}{*}{ Beheri improved } & \multirow[t]{3}{*}{$\mathbf{0}$} & $\mathbf{0}$ & 0.51 & 1.06 & 1.36 & 4.44 & 2.21 & 5.45 & 3.82 & 10.41 \\
\hline & & 2 & 0.36 & 1.19 & 1.89 & 3.83 & 3.08 & 4.70 & 5.15 & 9.12 \\
\hline & & 4 & 1.01 & 0.78 & 3.14 & 4.63 & 5.12 & 5.67 & 8.76 & 10.69 \\
\hline & \multirow[t]{3}{*}{30} & $\mathbf{0}$ & 0.70 & 0.90 & 2.09 & 4.73 & 3.41 & 5.81 & 5.85 & 10.99 \\
\hline & & 2 & 0.64 & 1.22 & 2.73 & 6.32 & 4.45 & 7.76 & 7.50 & 14.69 \\
\hline & & 4 & 0.67 & 1.26 & 2.94 & 5.80 & 4.78 & 7.12 & 8.05 & 13.55 \\
\hline & \multirow[t]{3}{*}{45} & $\mathbf{0}$ & 0.49 & 1.47 & 3.05 & 6.85 & 4.51 & 8.41 & 7.80 & 15.99 \\
\hline & & 2 & 0.48 & 1.53 & 3.39 & 8.76 & 5.53 & 10.74 & 9.16 & 20.26 \\
\hline & & 4 & 0.65 & 1.79 & 4.18 & 9.09 & 6.80 & 11.16 & 11.29 & 21.15 \\
\hline LSD at 0.05 level & & & 0.27 & 0.35 & 0.95 & 1.13 & 1.25 & 1.40 & 1.99 & 2.62 \\
\hline
\end{tabular}


Table 8. Effect of the triple interaction among cultivars, phosphorus rates and mycorrhizae levels on dry weight of different parts of onion plants at 75 and 105 days after transplanting during $2016 / 2017$ season

\begin{tabular}{|c|c|c|c|c|c|c|c|c|c|c|}
\hline \multirow{3}{*}{$\begin{array}{l}\text { Treatment } \\
\text { Cultivar }\end{array}$} & & & $\begin{array}{l}\text { Dry } \\
\text { roots }\end{array}$ & $\begin{array}{l}\text { eight of } \\
\text { /plant) }\end{array}$ & $\begin{array}{l}\text { Dry w } \\
\text { bulb ( }\end{array}$ & $\begin{array}{l}\text { eight of } \\
\text { g/plant) }\end{array}$ & $\begin{array}{l}\text { Dry we } \\
\text { leaves ( }\end{array}$ & $\begin{array}{l}\text { eight of } \\
\text { g/plant) }\end{array}$ & $\begin{array}{r}\text { Total dr } \\
(\mathrm{g} / \mathrm{p}\end{array}$ & $\begin{array}{l}\text { y weight } \\
\text { lant) }\end{array}$ \\
\hline & \multirow{2}{*}{$\begin{array}{c}\mathbf{P}_{2} \mathbf{O}_{5} \text { rates } \\
\text { (kg/fad.) }\end{array}$} & \multirow{2}{*}{$\begin{array}{l}\text { Mycorrhizae } \\
\text { level (kg/fad.) }\end{array}$} & \multicolumn{8}{|c|}{ Days after transplanting } \\
\hline & & & 75 & 105 & 75 & 105 & 75 & 105 & 75 & 105 \\
\hline \multirow[t]{9}{*}{ Giza 20} & $\mathbf{0}$ & $\mathbf{0}$ & 0.31 & 0.90 & 1.51 & 3.90 & 2.46 & 4.79 & 4.12 & 8.25 \\
\hline & & 2 & 0.56 & 0.86 & 1.75 & 3.51 & 2.86 & 4.30 & 4.89 & 7.44 \\
\hline & & 4 & 0.63 & 0.79 & 2.15 & 4.68 & 3.50 & 5.75 & 5.96 & 9.76 \\
\hline & 30 & $\mathbf{0}$ & 0.34 & 1.10 & 2.64 & 5.33 & 4.31 & 6.55 & 7.11 & 11.21 \\
\hline & & 2 & 0.49 & 1.58 & 3.07 & 4.65 & 5.01 & 5.71 & 8.32 & 10.09 \\
\hline & & 4 & 0.75 & 1.82 & 3.53 & 8.52 & 5.76 & 10.46 & 9.67 & 17.95 \\
\hline & 45 & $\mathbf{0}$ & 0.71 & 1.77 & 3.77 & 9.14 & 6.15 & 11.22 & 10.27 & 19.17 \\
\hline & & 2 & 0.75 & 2.24 & 3.66 & 10.88 & 5.97 & 13.36 & 10.01 & 22.88 \\
\hline & & 4 & 0.89 & 2.24 & 3.84 & 12.25 & 6.26 & 15.03 & 10.54 & 25.62 \\
\hline \multirow[t]{9}{*}{ Beheri improved } & $\mathbf{0}$ & $\mathbf{0}$ & 0.40 & 1.24 & 1.51 & 5.22 & 2.45 & 6.41 & 4.16 & 11.06 \\
\hline & & 2 & 0.40 & 1.40 & 2.25 & 4.50 & 3.67 & 5.53 & 6.11 & 9.70 \\
\hline & & 4 & 0.60 & 0.92 & 3.30 & 5.44 & 5.38 & 6.68 & 8.97 & 11.34 \\
\hline & 30 & $\mathbf{0}$ & 0.91 & 1.05 & 3.04 & 5.57 & 4.96 & 6.84 & 8.45 & 11.67 \\
\hline & & 2 & 0.85 & 1.44 & 4.04 & 7.43 & 6.59 & 9.13 & 11.05 & 15.58 \\
\hline & & 4 & 0.69 & 1.48 & 4.20 & 6.82 & 6.84 & 8.38 & 11.38 & 14.38 \\
\hline & 45 & $\mathbf{0}$ & 0.92 & 1.72 & 4.45 & 8.05 & 7.27 & 9.89 & 12.18 & 16.96 \\
\hline & & 2 & 0.83 & 1.80 & 4.56 & 10.30 & 7.45 & 12.64 & 12.42 & 21.49 \\
\hline & & 4 & 0.84 & 2.10 & 4.75 & 10.70 & 7.75 & 13.13 & 12.91 & 22.45 \\
\hline LSD at 0.05 level & & & 0.24 & 0.41 & 0.33 & 1.32 & 0.62 & 1.61 & 1.11 & 3.08 \\
\hline
\end{tabular}


Table 9. Effect of cultivars, phosphorus rates and mycorrhizae levels on nitrogen, phosphorus and potassium uptake $(\mathrm{mg})$ by different parts of onion at 105 days after transplanting during 2016/2017 season

\begin{tabular}{|c|c|c|c|c|c|c|c|c|c|}
\hline \multirow[t]{2}{*}{ Treatment } & \multicolumn{3}{|c|}{$\mathbf{N}$} & \multicolumn{3}{|c|}{$\mathbf{P}$} & \multicolumn{3}{|c|}{$\mathbf{K}$} \\
\hline & Roots & Bulb & Leaves & Roots & Bulb & Leaves & Roots & Bulb & $\overline{\text { Leaves }}$ \\
\hline & \multicolumn{9}{|c|}{ Effect of cultivars } \\
\hline Giza 20 & 24.91 & 122.38 & 252.54 & 5.18 & 25.27 & 40.26 & 32.60 & 158.57 & 212.55 \\
\hline Beheri improved & 23.24 & 118.17 & 238.71 & 4.46 & 22.28 & 38.60 & 27.84 & 143.31 & 196.07 \\
\hline \multirow[t]{2}{*}{ LSD at 0.05 level } & 1.26 & 2.52 & 3.89 & 0.13 & 0.80 & 0.08 & 1.67 & 2.73 & 2.50 \\
\hline & \multicolumn{9}{|c|}{ Effect of phosphorus rate (kg/fad.) } \\
\hline $\mathbf{0}$ & 16.69 & 79.58 & 157.23 & 3.47 & 15.88 & 25.34 & 20.54 & 96.26 & 131.19 \\
\hline 30 & 23.19 & 108.21 & 229.57 & 4.52 & 20.82 & 36.10 & 28.64 & 135.14 & 185.48 \\
\hline 45 & 32.33 & 173.04 & 350.07 & 6.47 & 34.62 & 56.85 & 41.48 & 121.42 & 296.25 \\
\hline \multirow[t]{2}{*}{ LSD at 0.05 level } & 0.84 & 2.45 & 6.28 & 0.08 & 0.53 & 0.57 & 0.62 & 2.83 & 5.74 \\
\hline & \multicolumn{9}{|c|}{ Effect of mycorrhizae levels (kg/fad.) } \\
\hline $\mathbf{0}$ & 20.79 & 103.68 & 215.75 & 4.34 & 21.23 & 34.43 & 26.91 & 135.85 & 178.99 \\
\hline 2 & 24.61 & 113.35 & 237.53 & 5.17 & 23.36 & 38.75 & 32.48 & 148.21 & 195.97 \\
\hline 4 & 26.83 & 143.80 & 283.59 & 4.95 & 26.73 & 45.11 & 31.27 & 168.76 & 237.97 \\
\hline LSD at 0.05 level & 0.71 & 2.58 & 5.52 & 0.07 & 0.47 & 0.54 & 0.73 & 2.75 & 5.05 \\
\hline
\end{tabular}

on garlic. They illustrated that there were a significant differences between garlic cultivars regarding to $\mathrm{N}, \mathrm{P}$ and $\mathrm{K}$ content, uptake and total uptake .

\section{Effect of phosphorus rates}

Nitrogen, phosphorus and potassium uptake by roots, bulb and leaves significantly increased with increasing $\mathrm{P}_{2} \mathrm{O}_{5}$ up to $45 \mathrm{~kg} / \mathrm{fad}$., at 105 days after transplanting in the second season only (Table 9).

The enhancing effect may be due to the essential and vital role of phosphorus that could stimulate root gridgeth as shown in Table 2, which promote the uptake by onion plant parts (Table 9).

These results are in harmony with those reported by Abd El-Fattah et al. (2002), Majumdar et al. (2003) and Mohsen (2012) on garlic. ElHadidi et al. (2016) and Júnior et al. (2016), showed that onion plants which fertilized with
$\mathrm{P}_{2} \mathrm{O}_{5}$ at $100 \mathrm{~kg} /$ fad., increased nitrogen, phosphorus, potassium and protein percentages in onion bulbs as well as nitrogen, phosphorus and potassium uptakes by plant parts.

\section{Effect of mycorrhizae colonization levels}

Mycorrhizae at 2 and $4 \mathrm{~kg} /$ fad., significantly increased N,P and $\mathrm{K}$ uptake by bulb and leaves compared to control treatment and mycorrhizae at $4 \mathrm{~kg} / \mathrm{fad}$., gave the highest value for each of $\mathrm{N}, \mathrm{P}$ and $\mathrm{K}$ uptake by bulb and leaves (Table 9), while each of $\mathrm{P}$ and $\mathrm{K}$ uptake by roots was the highest when plants inoculated with $2 \mathrm{~kg} / \mathrm{fad}$. mycorrhizae.

The obtained results are in agreement with those reported by Wani and Konde (1998), Sari et al. (2002), Gouda (2008) and Mohsen (2012) on garlic, Awad (2002) on potato. Shuab et al. (2014) found that chemical contents and their uptake by onion were the maximum with the plants infected with mycorrhizae as compared to the uninoculated ones. 
Effect of the interaction between cultivars and phosphorus rates

The interaction between cultivars and $\mathrm{P}_{2} \mathrm{O}_{5}$ had a significant effect on $\mathrm{N}, \mathrm{P}$ and $\mathrm{K}$ uptake by roots, bulb and leaves (Table 10). The interaction between Giza 20 cultivar and $\mathrm{P}_{2} \mathrm{O}_{5}$ at $45 \mathrm{~kg} / \mathrm{fad}$., gave the highest value for each of N,P and K uptake by roots, bulb and leaves.

These results coincide with those reported by Author et al. (1994) and Ortas (2008) on onion, Mohsen (2012) on garlic, Awad (2002) on potato. Abdullahi and Sheriff (2013) showed that inoculated onion plants with mycorrhizae fungi and fertilized with $60-30-50 \mathrm{~kg} / \mathrm{ha}$ NPK recorded the maximum concentration of $\mathrm{N}, \mathrm{P}$ and $\mathrm{K}$ contents in shoots than uninoculated plants with high rates (120-60-50 kg/ha NPK).

Effect of the interaction between cultivars and mycorrhizae levels

Results in Table 10 show that the interaction between Giza 20 cultivar and mycorrhizae at 4 $\mathrm{kg} /$ fad., increased N,P and $\mathrm{K}$ uptake by roots, bulb and leaves compared to the other interaction treatments at 105 days after transplanting in the second season only.
Effect of the interaction between phosphorus and mycorrhizae colonization levels

The obtained results in Table 11 indicate that the interaction between $\mathrm{P}_{2} \mathrm{O}_{5}$ and mycorrhizae had a significant effect on $\mathrm{N}, \mathrm{P}$ and $\mathrm{K}$ uptake by roots, bulb and leaves. The interaction between $45 \mathrm{~kg} /$ fad. $\mathrm{P}_{2} \mathrm{O}_{5}$ and $4 \mathrm{~kg} /$ fad. mycorrhizae gave the highest values.

In this regard, Ortas (2008) indicated that inoculation of the soil with mycorrhizae strains significantly increased $\mathrm{P}$ uptake in onion and garlic plants, especially under low $\mathrm{P}$ supply.

Effect of the triple interaction among cultivars, phosphorus and mycorrhizae colonization levels

Results in Table 12 indicate that, the triple interaction treatments among cultivars, $\mathrm{P}_{2} \mathrm{O}_{5}$ and mycorrhizae had a significant effect on N,P and $\mathrm{K}$ uptake by roots, bulb and leaves at 105 days after transplanting in the $2^{\text {nd }}$ season only. The interaction among Giza 20 cultivar, $\mathrm{P}_{2} \mathrm{O}_{5}$ at 45 $\mathrm{kg} /$ fad., and mycorrhizae at $4 \mathrm{~kg} /$ fad., increased $\mathrm{N}, \mathrm{P}$ and $\mathrm{K}$ uptake by roots, bulb and leaves.

Table 10. Effect of the interaction between cultivars and phosphorus and between cultivars and mycorrhizae on nitrogen, phosphorus and potassium uptake $(\mathrm{mg})$ by different parts of onion at 105 days after transplanting during 2016/2017 season

\begin{tabular}{|c|c|c|c|c|c|c|c|c|c|c|}
\hline \multirow[t]{2}{*}{ Treatment } & & \multicolumn{3}{|c|}{$\mathbf{N}$} & \multicolumn{3}{|c|}{$\mathbf{P}$} & \multicolumn{3}{|c|}{$\mathbf{K}$} \\
\hline & & Roots & Bulb & Leaves & Roots & Bulb & Leaves & Roots & Bulb & Leaves \\
\hline$\overline{\text { Cultivar }}$ & $\begin{array}{c}\mathbf{P}_{2} \mathbf{O}_{5} \\
\text { (kg/fad.) }\end{array}$ & \multicolumn{9}{|c|}{ Effect of the interaction between cultivars and phosphorus rates } \\
\hline \multirow[t]{3}{*}{ Giza 20} & 0 & 14.87 & 74.54 & 144.11 & 2.98 & 14.62 & 22.32 & 19.03 & 93.91 & 123.27 \\
\hline & 30 & 26.24 & 112.05 & 226.03 & 5.13 & 21.55 & 35.39 & 32.49 & 139.05 & 187.48 \\
\hline & 45 & 33.61 & 180.56 & 387.47 & 7.43 & 39.64 & 63.06 & 46.29 & 242.75 & 326.91 \\
\hline \multirow[t]{3}{*}{ Beheri improved } & $\mathbf{0}$ & 18.52 & 84.62 & 170.34 & 3.96 & 17.14 & 28.37 & 22.04 & 98.61 & 139.12 \\
\hline & 30 & 20.15 & 104.38 & 233.12 & 3.91 & 20.10 & 36.81 & 24.79 & 131.23 & 183.49 \\
\hline & 45 & 31.06 & 165.51 & 312.67 & 5.52 & 29.60 & 50.63 & 36.67 & 200.09 & 265.59 \\
\hline \multicolumn{2}{|l|}{ LSD at 0.05 level } & 1.19 & 3.47 & 8.89 & 0.12 & 0.75 & 0.81 & 0.88 & 4.00 & 7.74 \\
\hline Cultivar & (kg/fad.) & \multicolumn{9}{|c|}{ Effect of the interaction between cultivars and mycorrhizae levels } \\
\hline \multirow[t]{3}{*}{ Giza 20} & $\mathbf{0}$ & 21.25 & 108.29 & 221.16 & 4.41 & 22.16 & 34.52 & 28.38 & 142.82 & 185.63 \\
\hline & 2 & 25.32 & 106.28 & 227.96 & 5.54 & 23.14 & 36.52 & 34.67 & 143.64 & 190.59 \\
\hline & 4 & 28.15 & 152.58 & 308.49 & 5.59 & 30.51 & 49.73 & 34.76 & 189.25 & 261.43 \\
\hline \multirow[t]{3}{*}{ Beheri improved } & $\mathbf{0}$ & 20.32 & 99.07 & 210.33 & 4.27 & 20.30 & 34.34 & 25.45 & 128.88 & 172.34 \\
\hline & 2 & 23.90 & 120.42 & 247.11 & 4.80 & 23.58 & 40.97 & 30.28 & 152.78 & 201.35 \\
\hline & 4 & 25.50 & 135.02 & 258.69 & 4.32 & 22.95 & 40.49 & 27.78 & 148.28 & 214.51 \\
\hline LSD at 0.05 level & & 1.00 & 3.65 & 7.81 & 0.10 & 0.66 & 0.76 & 1.04 & 3.89 & 7.14 \\
\hline
\end{tabular}


Table 11. Effect of the interaction between phosphorus rates and mycorrhizae levels on nitrogen, phosphorus and potassium uptake $(\mathrm{mg})$ by different parts of onion at 105 days after transplanting during 2016/2017 season

\begin{tabular}{ccccccccccc}
\hline Treatment & & \multicolumn{3}{c}{$\mathbf{N}$} & \multicolumn{3}{c}{$\mathbf{P}$} & \multicolumn{5}{c}{ K } \\
\cline { 2 - 11 } & & Roots & Bulb & Leaves & Roots & Bulb & Leaves & Roots & Bulb & Leaves \\
\hline $\begin{array}{c}\mathbf{P}_{\mathbf{2}} \mathbf{O}_{\mathbf{5}} \text { rate } \\
\text { (kg/fad.) }\end{array}$ & $\begin{array}{c}\text { Mycorrhizae } \\
\text { level (kg/fad.) }\end{array}$ & & & & & & & & & \\
\hline $\mathbf{0}$ & $\mathbf{0}$ & 16.82 & 77.75 & 161.02 & 3.84 & 16.96 & 25.40 & 20.93 & 95.59 & 129.35 \\
& $\mathbf{2}$ & 18.19 & 70.58 & 139.97 & 3.91 & 14.15 & 22.70 & 23.20 & 85.04 & 117.31 \\
& $\mathbf{4}$ & 15.07 & 90.40 & 170.69 & 2.66 & 16.52 & 27.94 & 17.48 & 108.15 & 146.91 \\
$\mathbf{3 0}$ & $\mathbf{0}$ & 17.32 & 90.17 & 183.78 & 3.34 & 17.24 & 30.38 & 22.69 & 120.13 & 156.83 \\
& $\mathbf{2}$ & 24.35 & 99.26 & 214.99 & 4.88 & 19.67 & 35.17 & 30.79 & 124.62 & 175.53 \\
& $\mathbf{4}$ & 27.90 & 135.22 & 289.95 & 5.35 & 25.55 & 42.74 & 32.44 & 160.67 & 224.09 \\
\multirow{4}{*}{$\mathbf{4 5}$} & $\mathbf{0}$ & 28.22 & $143.12 \mathrm{c}$ & 302.44 & 5.83 & 29.48 & 47.52 & 37.12 & 191.82 & 250.79 \\
& $\mathbf{2}$ & 31.28 & 170.21 & 357.63 & 6.73 & 36.26 & 58.37 & 43.44 & 234.97 & 295.05 \\
& $\mathbf{4}$ & 37.50 & 205.78 & 390.15 & 6.86 & 38.11 & 64.65 & 43.89 & 237.47 & 342.91 \\
\multirow{2}{*}{ LSD at 0.05 level } & & 1.23 & 2.48 & 9.57 & 0.12 & 0.81 & 0.94 & 1.27 & 4.76 & 8.75 \\
\hline
\end{tabular}

Table 12. Effect of the triple interaction among cultivars, phosphorus rates and mycorrhizae levels on nitrogen, phosphorus and potassium uptake $(\mathrm{mg})$ by different parts of onion at 105 days after transplanting during 2016/2017 season

\begin{tabular}{|c|c|c|c|c|c|c|c|c|c|c|c|}
\hline \multicolumn{3}{|l|}{ Treatment } & \multicolumn{3}{|c|}{$\mathbf{N}$} & \multicolumn{3}{|c|}{$\mathbf{P}$} & \multicolumn{3}{|c|}{$\mathbf{K}$} \\
\hline$\overline{\text { Cultivar }}$ & $\begin{array}{c}\mathrm{P}_{2} \mathrm{O}_{5} \text { rate } \\
\text { (kg/fad.) }\end{array}$ & $\begin{array}{l}\text { Mycorrhizae } \\
\text { level (kg/fad.) }\end{array}$ & Roots & Bulb & Leaves & Roots & Bulb & Leaves & Roots & Bulb & Leaves \\
\hline \multirow[t]{9}{*}{ Giza 20} & $\mathbf{0}$ & $\mathbf{0}$ & 15.00 & 70.07 & 136.36 & 3.14 & 14.43 & 21.18 & 20.01 & 89.57 & 116.40 \\
\hline & & 2 & 15.10 & 65.40 & 132.30 & 3.05 & 12.80 & 19.63 & 19.57 & 82.49 & 109.65 \\
\hline & & 4 & 14.51 & 88.14 & 163.68 & 2.76 & 16.64 & 26.16 & 17.51 & 109.67 & 143.75 \\
\hline & 30 & $\mathbf{0}$ & 19.43 & 97.89 & 174.45 & 3.97 & 19.45 & 29.23 & 24.42 & 123.48 & 161.13 \\
\hline & & 2 & 26.96 & 82.62 & 163.69 & 5.61 & 16.89 & 26.83 & 34.65 & 105.09 & 145.03 \\
\hline & & 4 & 32.33 & 155.63 & 339.95 & 5.81 & 28.31 & 50.10 & 38.40 & 188.58 & 256.27 \\
\hline & 45 & $\mathbf{0}$ & 29.32 & 156.90 & 352.68 & 6.11 & 32.59 & 53.14 & 40.71 & 215.40 & 279.38 \\
\hline & & 2 & 33.89 & 170.82 & 387.89 & 7.96 & 39.74 & 63.10 & 49.80 & 243.35 & 317.08 \\
\hline & & 4 & 37.63 & 213.97 & 421.84 & 8.21 & 46.59 & 72.94 & 48.38 & 269.50 & 384.27 \\
\hline \multirow[t]{9}{*}{ Beheri improved } & $\mathbf{0}$ & $\mathbf{0}$ & 18.64 & 85.43 & 185.68 & 4.55 & 19.50 & 29.61 & 21.86 & 101.62 & 142.30 \\
\hline & & 2 & 21.28 & 75.75 & 147.65 & 4.77 & 15.51 & 25.77 & 26.83 & 87.60 & 124.98 \\
\hline & & 4 & 15.64 & 92.66 & 177.69 & 2.56 & 16.41 & 29.72 & 17.44 & 106.62 & 150.08 \\
\hline & 30 & $\mathbf{0}$ & 15.22 & 82.44 & 193.12 & 2.71 & 15.03 & 31.53 & 20.96 & 116.78 & 152.53 \\
\hline & & 2 & 21.74 & 115.91 & 266.29 & 4.14 & 22.46 & 43.52 & 26.92 & 144.14 & 206.03 \\
\hline & & 4 & 23.48 & 114.80 & 239.95 & 4.88 & 22.80 & 35.39 & 26.49 & 132.76 & 191.90 \\
\hline & 45 & $\mathbf{0}$ & 27.11 & 129.34 & 252.20 & 5.55 & 26.37 & 41.90 & 33.54 & 168.24 & 222.20 \\
\hline & & 2 & 28.68 & 169.61 & 327.38 & 5.50 & 32.78 & 53.63 & 37.08 & 226.60 & 273.02 \\
\hline & & 4 & 37.38 & 197.59 & 358.45 & 5.50 & 29.63 & 56.37 & 39.41 & 205.44 & 301.55 \\
\hline LSD at 0.05 level & & & 1.74 & 6.33 & 13.54 & 0.17 & 1.15 & 1.33 & 1.80 & 6.74 & 12.37 \\
\hline
\end{tabular}




\section{Conclusion}

From foregoing results, it could be concluded that, the triple interaction among Giza 20 cultivar and fertilized onion plants with $\mathrm{P}_{2} \mathrm{O}_{5}$ at $45 \mathrm{~kg} / \mathrm{fad}$., and colonization with mycorrhiza at $4 \mathrm{~kg} / \mathrm{fad}$., was the superior treatment for enhancing onion plant dry weight and N,P and $\mathrm{K}$ uptake by different parts of onion than other treatments.

\section{REFERENCES}

Abd El-Fattah, A.E., Z.S. El-Shal and E.M. Awad (2002). Response of garlic productivity and storability to application times of phosphorus and certain micro nutrients. J. Agric. Sci. Mansoura Univ., 27 (9): 62316241.

Abdullahi, R. and H.H. Sheriff (2013). Effect of Arbuscular mycorrhizal fungi and chemical fertilizer on gridgeth and shoot nutrients content of onion under field condition in Northern Sudan Savanna of Nigeria. J. Agric. Vet. Sci., 3 (5): 85-90

Abo El-Hamd, A.S.A., M.T. El-Abd, A.A.M. Mohamed and M.G. Zein El-Din (2016). Effect of some agricultural treatments on productivity and quality of green onion for export (Allium cepa L.). Middle East J. Agric. Res., 5 (1): 37-44.

Abou El-Magd, M.M., H.A. Mohamed and M.M.H. Abd El-Baky (2004). Organic and mineral fertilization of onion crop in relation to its vegetative gridgeth, yield and bulb quality. Egypt. J. Appl. Sci., 19 (7A): 265- 278.

Afek, U., J.A. Menge and E.L.V. Jonson (1991). Interaction among mycorrhizae, soil solarization, metalaxyl and plants in the field. Plant Disease, 75 (7): 665 - 671.

Author, S., C.R. Kshirsajar, V.K. Mandhare, H.B. Kalbhor and P.L. Patil (1994). Response of onion to Azotobacter and VA-mycorrhizal inoculation along with phosphorus levels. J. Maharashtra Agric. Univ., 19 (3): 476-477.

Awad, E.M. (2002). Gridgeth, yield and quality of potato crop as affected by the inoculation with vesicular arbuscular mycorrhizal (VAM) fungi under different levels of phosphorus. J. Agric. Sci. Mansoura Univ., 27 (8): 5593-5605.

Bolandnazar, S. (2009). The effect of mycorrhizal fungi on onion (Allium cepa L.) gridgeth and yield under three irrigation intervals at field condition. J. Food, Agric. Environ., 7 (2) : $360-362$.

Bremner, J.M. and C.S. Mulvaney (1982). Total nitrogen. In: Page, A.L., R.H. Miller and D.R. Keeney (Eds). Methods of Soil Analysis. Part 2, Ame. Soc. Agron. Madison, WI. USA, 595 - 624.

El-Desuki, M. (2004). Response of onion plants to humic acid and mineral fertilizers application. Ann. Agric. Sci. Moshtohor, 42 (4): 1955- 1964.

El-Hadidi, E.M., M.M. El-Shazly and H.M.M. Hegazy (2016). Effect of $\mathrm{N}, \mathrm{P}$ and $\mathrm{Cu}$ fertilization on onion yield, quality and nutrients uptake. J. Soil Sci. and Agric. Eng., Mansoura Univ., 7(2): $231-236$.

El-Hamady, M.M. (2017). Gridgeth and yield of onion (Allium cepa L.) as influenced by nitrogen and phosphorus fertilizers levels. Canadian J. Agric. and Crops, 2 (1) : 34-41.

El-Kalla, S.E., A.K. Mostafa, A.A. Leilah and A. Awad-Rokia (1997). Mineral and biophosphatic fertilization for intercropped faba bean and onion. Egypt. J. Agric. Res., 77 (1): $253-271$.

El-Morsy, A.H.A., A.E.A. Abdel-Fattah and Z.S.A. El-Shal (2002). Effect of phosphate fertilizer and VA-Mycorrhizal inoculation on gridgeth, tuber yield and quality of sweet potato. Proc. Minia First Conf. Agric. Environ. Sci. Minia Egypt. March 25-28, $1815-1827$.

El-Mansi, A.A., M.A. I.Khalil, A.A Gad, M.H. El-Sawah, and M.A. Zaki. (1984). Effect of potassium fertilizer on the productivity and storageability of garlic. Zagazig J. Agric. Res., 12 (2): 182-193.

El-Sheekh, H.M. (1997). Effect of bio and mineral phosphate fertilizers on gridgeth, yield, quality and storability of onion. Egypt J. Appl. Sci., 12 (12): 213-231. 
Gosling, P., A. Hodge, G. Goodlass, and G.D. Bending (2006). Arbuscular mycorrhizal fungi and organic farming. Agric., Ecos. and Environ., 113 : 17-35.

Gouda, A.E.D. (2008). Effect of some nitrogenous and phosphatic fertilizers sources and VAmycorrhiza inoculum on gridgeth, productivity and storability of garlic (Allium sativum L.). $\mathrm{Ph}$. D. Thesis, Fac. Agric., Mansoura Univ., Egypt.

Jackson, M.L. (1970). Soil Chemical Analysis Prentice Hall. Englewood Gliffs, N.J.

Júnior, J.N., R.M.P. Ribeiro, A.P. Chaves, V.F.L. Sousa, L.C. Grangeiro, M.Z. Negreiros, S.P. Marrocos and G.S.O. Rodrigues (2016). Effect of phosphorus fertilization on yield and quality of onion bulbs. Afr. J. Agric. Res., 11 (45): 4594-4599.

Kandil, A.A., A.A. Leilah, A.Kh. Mostafa and F.H.F. Hassan (2010). Gridgeth, yield and storability of onion bulbs, as affected by irrigation treatments and cultivars referring to water consumption. J. Pl. Prod., 1 (2): 193 $-204$.

Kandil, A.A., A.E. Sharief and F.H. Fathalla (2013). Effect of transplanting dates of some onion cultivars on vegetative gridgeth, bulb yield and its quality. Esci. J. Crop Prod., 2 (3) : 72-82.

Kishor, S., R.B. Ram, S. Kishor, M.L. Meena and S. Kumar (2017). Effect of spacing and different cultivars on gridgeth and yield of onion (Allium cepa L.) under Lucknow conditions. Int. J. Pure Appl. Biosci., 5 (4): 612-616.

Mahmoud, H.A.F. and E.M. El-Hefny (1999). Effect of mycorrhizal infection and phosphorus concentrations on onion plant. Ann. Agric. Sci. Moshtohor, 37 (3): 18051817.

Mahmoud, H.A.F., F.A. Abo Sedera and S.B.D. Yousef (2000). Effect of organic and inorganic fertilizers on onion crop. J. Agric. Sci., Mansoura Univ., 25 (9): 5813 - 5829.

Majumdar, B., M.S. Venkatesh, K. Kumar and V. Patiram (2003). Response of garlic (Allium sativum L.) to phosphorous and sulphur application in acid alfisol of Meghalaya. J. Spices Aromatic Crops, 12 (2): 183-186.

Marschner, H. (1995). Mineral Nutrition of Higher Plants. $2^{\text {nd }}($ Ed.), Academic Press Limeted, Text Book. London, England.

Mohamed H.M. (2015). Impact of inoculation with Arbuscular Mycorrhizal, phosphate solubilizing bacteria and soil yeast on gridgeth, yield and phosphorus content of onion plants. Int. J. Soil Sci., 10: 93-99.

Mohsen, A.A.M. (2012). Response of garlic plant to nitrogen, phosphorus, potassium and some biofertilizer levels under sandy soil conditions. Ph.D. Thesis, Fac. Agric. Zagazig Univ., Egypt.

Muddathir, A.M. (2007). Effect of Vesicular Arbuscular Mycorrhizal (VAM) inoculation and phosphorus treatments on gridgeth and yield of three onions (Allium cepa L.) cultivars. M.Sc. Thesis, Fac. Agric. Khartoum Univ., Sudan.

Olsen, S.R. and L.E. Sommers. (1982). Phosphorus. In: Page, A.L., R.H. Miller and D.R. Keeney (eds). Methods of Soil Analysis. Part 2, Ame. Soc. Agron. Madison, W.I. USA, 403 - 430.

Ortas, I. (2008). The effect of mycorrhizal inoculation on forage and non-forage plant gridgeth and nutrient uptake under field conditions. Options Mediterraneennes- Series A, $79: 463-469$.

Pacovsky, R.S. and G. Fuller (1986). Development of two endomycorrhizal symbioses on soybean and comparison with phosphorus fertilization. Plant and Soil, 195 : 361-377.

Patel, Z.G. and M.U. Vachhani. (1994). Effect of NPK fertilization on the yield and quality of onion. Hort. J., 7 (1): 75.

Ristimuki, L.M., I. Papadopoulos, C. Sannwel, and N.J. Berhoyen, (2000). Slow release fertilizers on vegetables. Acta Hort., 511: 125-131.

Sari, N., I. Ortas and H. Yetisir (2002). Effect of mycorrhizae inoculation on plant gridgeth, 
yield and phosphorus uptake in garlic under field conditions. Communications in Soil Sci. and Pl. Anal., 33 (13/14) : 2189 - 2201 .

Shaheen, A.M., F.A. Rizk and S.M. Singer (2007). Gridgeing onion plants without chemical fertilization. Res. J. Agric. Bio., Sci., 3 (2): 95 - 104.

Sharma, M.P., A. Adholeya and A. Adholeya (2000). Enhanced gridgeth and productivity following inoculation with indigenous AM fungi in four varieties of onion (Allium cepa L.) in an alfisol. Bio. Agric. Hort., 18 (1): 1427.

Shinde, S.K. and B.P. Shinde (2016). Consequence of Arbuscular mycorrhiza on enhancement, gridgeth and yield of onion (Allium cepa L.). Int. J. Life. Sci. Scienti. Res., 2 (2): 206-211

Shuab, R., R. Lone, J. Naidu, V. Sharma, S. Imtiyaz and K.K. Koul. (2014). Benefits of inoculation of Arbuscular mycorrhizal fungi on gridgeth and development of onion (Allium cepa) plant. Ame. Eurasian J. Agric. Environ. Sci., 14 (6): 527-535.

Singh, A., S. Singh and B. Singh (2002). Effect of VAM and inorganic fertilizers on gridgeth and yield of onion (Allium cepa L.). Veg. Sci., 29 (1): 40-42.

Smith, S.E. and D.J. Read (1997). Mycorrhizal Symbiosis. Academic Press, London.

Snedecor, G.W. and W.G. Cochran (1980). Statistical Methods. $7^{\text {th }}$ Ed. Iowa State Univ. Press, Ames. Iowa, USA.

Tao Guo, J., C. Zunling and L. Xiaolin (2006). Effect of Arbuscular mycorrhizal fungi and ammonium: nitrate on gridgeth and pungency of seedlings. J. Pl. Nutr., 29:1047-1059.

Wani, P.V. and B.K. Konde (1998). Effects of Glomus mosseae inoculation using different P-sources on garlic. J. Maharashtra Agric. Univ., 23 (1): 39 - 42.

\section{تأثير التسميد القوسفاتى والتلقيح بالميكورهيزا على الوزن الجاف والمحتوى الكيماوى النباتات صنفين من البصل}

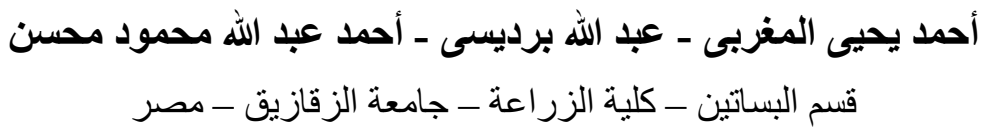

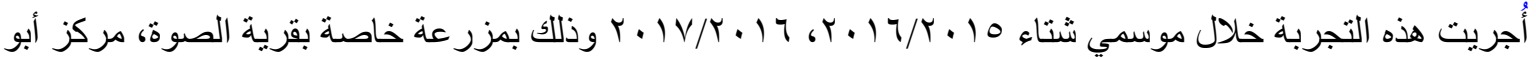

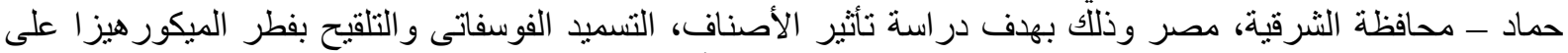

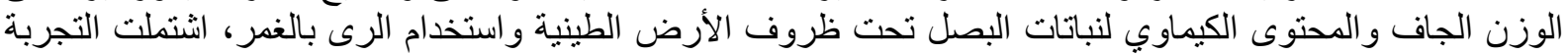

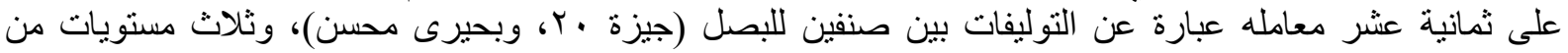

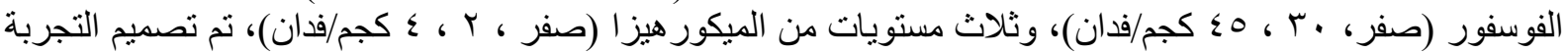

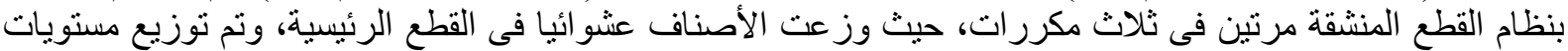

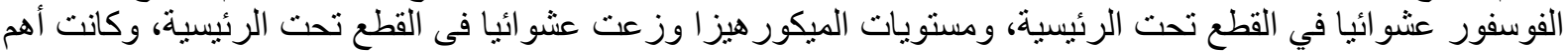

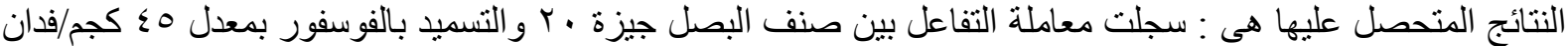

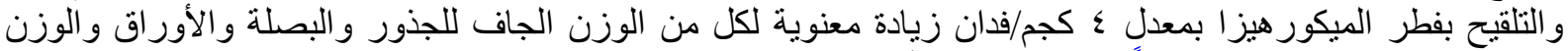

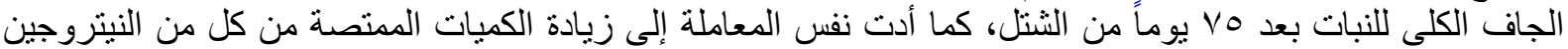

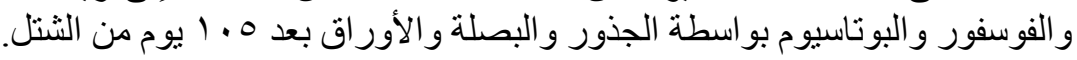

